SLAC - PUB - 4695

August 1988

( $\mathrm{T})$

\title{
BRST QUANTIZATION OF THE CP ${ }^{1}$ MODEL WITH CHERN-SIMONS TERM IN 2+1 DIMENSIONS*
}

\author{
PURUSHOTHAM VORUGANTI \\ Stanford Linear Accelerator Center, \\ Stanford University, Stanford, California 94309 \\ and \\ Department of Physics, \\ Stanford University, Stanford, California 94305
}

\begin{abstract}
Field theory on $\mathrm{CP}^{1}$ with Chern-Simons term is analyzed classically and then quantized. The constrained system is disentangled using the elegant BRST method. The quantum Hamiltonian, the BRST charge and the anti-BRST charge are derived. Application to a recently proposed model with principal chiral fields is elaborated.
\end{abstract}

Submitted to Physical Review D

*Work supported by the Department of Energy, contract DE-AC03-76SF00515. 


\section{Introduction}

The $\mathrm{CP}^{1}$ model $^{1}$ is an attractive starting point to analyze the physics of the nonlinear sigma model. The model exhibits solitons, Hopf instantons, and novel spin and statistics in $2+1$ space-time dimensions with inclusion of the ChernSimons term. ${ }^{2}$ As numerous authors have now shown, at special values of the coefficient for the Chern-Simons term, bose-fermi transmutation ensues. These disparate phenomena and their consequences may find their test in the twodimensional anti-ferromagnets from which high- $T_{c}$ superconductors are thought to arise. ${ }^{3}$ This is the motivation for understanding the canonical structure of this field theory model.

Most generally, the model has the following Lagrangian density:

$$
\mathcal{L}=\left(D_{\mu} Z\right)^{\dagger}\left(D^{\mu} Z\right)-\lambda\left(Z^{\dagger} Z-1\right)+\theta \varepsilon^{\mu \nu \lambda} A_{\mu} \partial_{\nu} A_{\lambda}
$$

where $D_{\mu}=\left(\partial_{\mu}+i A_{\mu}\right)$ and $Z$ is a complex two-spinor $\left(Z_{1}, Z_{2}\right)$. The Lagrangian has the symmetry $\mathrm{SU}(2)_{\text {global }} \times \mathrm{U}(1)_{\text {local }}$. Obtaining the correct canonical structure within the Hamiltonian formulation serves two basic purposes. First, the physical Hilbert space and the corresponding time evolution operator in the constrained phase space can be obtained. Second, it will serve as the starting point for calculation of quantum effects either through a canonical perturbation series or through lattice regularized methods, or if permissible, a path integral approach. The Hamiltonian structure is not at all trivial. The system has two constraint fields, namely the $\lambda(x)$ field and the time component of the gauge field $A_{0}(x)$. These lead to a number of primary and secondary constraints. Various workers have derived the canonical structure of the nonlinear sigma model ${ }^{4}$ and the $\mathrm{CP}^{1}$ model $^{5}$ with or without the Hopf term (versus the Chern-Simons term).

_ Most have quantized either through the traditional method of $\operatorname{Dirac}^{6}$ in presence of the second class constraints or simply solved for the constraints and started with a new effective Lagrangian. These routes usually lead to non-canonical commutation relations and the physical subspace of Hilbert space is not always lucid. 
We will exploit the elegance and clarity of the BRST method ${ }^{7}$ to quantize the above field theory and obtain in this way a canonical hamiltonian with canonical commutation and anti-commutation relations and a simply defined physical Hilbert space. The resulting theory represents exactly the constrained system in a particular gauge. The price one pays is the additional degrees of freedom like the BRST ghost degrees of freedom introduced in the process (which decouple) and a nonlinear Hamiltonian. We will follow the work of Nemeschansky, Preitschopf and Weinstein (NPW) for the application at hand. ${ }^{8}$ The outline of the paper is roughly as follows: in Sec. II, we present a classical analysis of the problem and derive an effective Lagrangian in a particular gauge; in Sec. III, we attempt a canonical quantization and elaborate the various. constraints that arise; in Sec. IV, we adopt the BRST method and quantize the system in this framework; in Sec. V, we analyze the same system after making a map using principal $\mathrm{SU}(2)$ matrices and derive the path integral action for this theory; in Sec. VI, we summarize and make a few conclusions.

\section{Classical Effective Lagrangrian}

In this section we will eliminate the gauge degrees of freedom entirely, thus obtaining a new classical Lagrangian. The equations of motion that result from (1) are

$$
\begin{aligned}
\left(D_{\mu} D^{\mu}+\lambda\right) Z & =0 \\
Z^{\dagger} Z & =1 \\
A_{\mu} Z^{\dagger} Z & =-\frac{J_{\mu}}{2}-\theta \varepsilon_{\mu \nu \lambda} \partial^{\nu} A^{\lambda},
\end{aligned}
$$

where $J_{\mu}=i\left(\partial_{\mu} Z^{\dagger} Z-Z^{\dagger} \partial_{\mu} Z\right)$. We can solve the second constraint equation using any convenient parametrization of the $Z(x)$ fields. For example, we can write $Z=\left(Z_{1}{ }^{\prime}, Z_{2}{ }^{\prime}\right)$ where

$$
\left(Z_{1}^{\prime}, Z_{2}^{\prime}\right)=\frac{1}{\sqrt{Z^{\dagger} Z}}\left(Z_{1}, Z_{2}\right)
$$


Therefore, the $\lambda(x)$ constraint field is unnecessary. The $\mu=0$ component of the third equation of motion is actually a constraint which, when using $Z^{\dagger} Z=1$, becomes

$$
A_{0}=-\frac{J_{0}}{2}-\theta \varepsilon_{i j} \partial^{i} A^{j}
$$

The theory is $\mathrm{U}(1)$ gauge invariant, thus we make the gauge choice $A_{0}=0$. Now the remaining gauge degrees of freedom can most generally be expressed as

$$
A_{i}=\partial_{i} \phi+\varepsilon_{i j} \partial_{j} \psi
$$

where $\psi(x)$ and $\phi(x)$ are some scalar functions. Equation (3) now means we can solve for $\psi(x, t)$ :

$$
\psi(x, t)=\frac{1}{2 \theta} \int d^{2} x^{\prime} D\left(x-x^{\prime}\right) J_{0}\left(x^{\prime}, t\right)
$$

where $D\left(x-x^{\prime}\right)$ solves $\partial^{2} D\left(x-x^{\prime}\right)=\delta^{(2)}\left(x-x^{\prime}\right)$ and is given by

$$
D\left(x-x^{\prime}\right)=\frac{1}{4 \pi} \ln \left(x-x^{\prime}\right)^{2}+\text { const } .
$$

The covariant (Noether) current is given by $J_{\text {noether }}^{\mu}=i\left(D^{\mu} Z^{\dagger} Z-Z^{\dagger} D^{\mu} Z\right)$. By taking the derivative of the third equation of motion, we find $\partial_{\mu} J_{\text {noether }}^{\mu}=0$. This implies, since $Z^{\dagger} Z=1$,

$$
\partial_{i} A^{i}=-\frac{1}{2} \partial_{\mu} J^{\mu}
$$

where $i=1,2$ and $\mu=0,1,2$. This implies a solution for $\phi(x, t)$ :

$$
\phi(x, t)=-\frac{1}{2} \int d^{2} x^{\prime} D\left(x-x^{\prime}\right) \partial_{\mu} J^{\mu}\left(x^{\prime}, t\right)
$$

Putting all this together, we can re-express the Lagrangian in terms of the con-strained $Z(x)$ fields in the $A_{0}=0$ gauge:

$$
\mathcal{L}_{e f f}=\left(\partial_{\mu} Z\right)^{\dagger}\left(\partial^{\mu} Z\right)-A_{i} J_{i}-A_{i} A_{i}-\theta \varepsilon_{i j} A_{i} \dot{A}_{j},
$$


where again $i=1,2$ and the dot implies a time derivative. For the boundary condition at spatial and temporal infinity $A_{i}(\infty)=0$, the last term can be written as $-\theta\left(\partial^{2} \phi \dot{\psi}-\partial^{2} \psi \dot{\phi}\right)$. Alternatively, the third equation of motion implies

$$
A_{\mu} A^{\mu} Z^{\dagger} Z=-\frac{A_{\mu} J^{\mu}}{2}-\theta \varepsilon^{\mu \nu \lambda} A_{\mu} \partial_{\nu} A_{\lambda}
$$

which implies for the effective action

$$
\mathcal{L}_{\text {eff }}=\left(\partial_{\mu} Z\right)^{\dagger}\left(\partial^{\mu} Z\right)-\frac{1}{2} J_{i} A_{i}
$$

Now substituting the expressions for $A_{i}, \phi(x), \psi(x)$ from Eqs. (4), (5) and (8), we have completely eliminated the gauge degrees of freedom. The effective Lagrangian is admittedly difficult to quantize because of the various non-local interactions but not impossible. The fact that we could eliminate both $A_{1}$ and $A_{2}$ was unexpected and it depends crucially on using fields $Z(x)$ constrained to modulus one. It would be desirable to quantize the theory without recourse to any classical equations of motion. In the next section, instead of eliminating any degrees of freedom, we will elaborate the complexity of the various constraints hidden in the above approach which side-stepped them.

\section{Canonical Quantization and Constraints}

In outlining the traditional method of quantization, we will derive the canonical momenta for all the fields and hence the Hamiltonian. In this process, we will find some primary constraints. Requiring the time variation of these constraints, i.e., the commutator with the Hamiltonian, to vanish will introduce secondary constraints and so on. The Lagrangian density in Eq. (1) implies for the canonical field momenta:

$$
\begin{aligned}
& \Pi_{z}=\left(D_{0} Z\right)^{\dagger} \quad \Pi_{A_{1}}=\theta A_{2} \quad \Pi_{A_{0}}=0 \\
& \Pi_{z^{\dagger}}=\left(D_{0} Z\right) \quad \Pi_{A_{2}}=-\theta A_{1} \quad \Pi_{\lambda}=0 .
\end{aligned}
$$


The two equations in the last column are typical primary constraints. The middle two equations imply $A_{1}(x)$ and $A_{2}(x)$ are conjugate to each other. This is obvious had we added a total time derivative $\theta \partial_{t}\left(A_{1} A_{2}\right)$ to the Lagrangian density. This eliminates time derivatives of $A_{2}$ giving $\Pi_{A_{1}}=2 \theta A_{2}$ and $\Pi_{A_{2}}=0$. $A_{2}(x)$ can now be eliminated entirely in favor of $\Pi_{A_{1}}$ and the constraint $\Pi_{A_{2}}=0$ is exactly zero for all times. For simplicity, we will use $A(x)$ for $A_{1}(x)$ and $\Pi_{A}(x)$ for $\Pi_{A_{1}}(x)$. Therefore, we rewrite the Lagrangian density in terms of the new variables:

$$
\mathcal{L}=\Pi_{z} \dot{Z}+\dot{Z}^{\dagger} \Pi_{z^{\dagger}}+\Pi_{A} \dot{A}-\not{H}
$$

where the Hamiltonian density is

$$
\begin{aligned}
\mathcal{H} & =\Pi_{z} \Pi_{z^{\dagger}}+\left(\partial_{i} Z\right)^{\dagger}\left(\partial_{i} Z\right)+A J_{1}+\frac{1}{2 \theta} \Pi_{A} J_{2}+\left(A A+\frac{1}{4 \theta^{2}} \Pi_{A} \Pi_{A}\right) Z^{\dagger} Z \\
& -A_{0}\left(i \Pi_{z} Z-i Z^{\dagger} \Pi_{z^{\dagger}}+\partial_{1} \Pi_{A}-2 \theta \partial_{2} A\right)+\lambda\left(Z^{\dagger} Z-1\right) .
\end{aligned}
$$

We note that the Hamiltonian has no $\Pi_{A}^{2}$ term as one would expect for usual dynamical gauge fields. The Hamiltonian equations of motion are derived using the canonical equal-time commutation relations

$$
\begin{aligned}
{\left[Z(x), \Pi_{z}\left(x^{\prime}\right)\right] } & =\left[Z^{\dagger}(x), \Pi_{z^{\dagger}}\left(x^{\prime}\right)\right]=\left[\lambda(x), \Pi_{\lambda}\left(x^{\prime}\right)\right]=\left[A_{0}(x), \Pi_{A_{0}}\left(x^{\prime}\right)\right] \\
& =\left[A(x), \Pi_{A}\left(x^{\prime}\right)\right]=i \delta^{2}\left(x-x^{\prime}\right)
\end{aligned}
$$

and the operator relation for time evolution $\dot{\mathcal{O}}=i[H, 0]$ where $H=\int d^{2} x \mathcal{H}(x)$. It is easily confirmed that the Lagrange equations of motion found in Sec. II are exactly reproduced. As required, the $A(x)$ equation of motion is the $A_{1}(x)$ equation of motion and the $\Pi_{A}(x)$ equation of motion is the $A_{2}(x)$ equation of motion. The two trivial equations of motion $\dot{\lambda}=0$ and $\dot{A}_{0}=0$ are correct for the Lagrange multipliers. Requiring $\dot{\Pi}_{A_{0}}=0$ gives

$$
i \Pi_{z} Z-i Z^{\dagger} \Pi_{z^{\dagger}}+\partial_{1} \Pi_{A}-2 \theta \partial_{2} A=0
$$

With the expressions for the canonical momenta, this secondary constraint is exactly the $A_{0}$ equation of motion. In fact, this secondary constraint is the 
generator for time independent gauge transformations. Gauge-invariant physical states should be annihilated by this constraint operator. Physical states will be simultaneous eigenstates of the Hamiltonian and the constraint only if the two commute. Indeed, after some algebra, one confirms $\left[H, C_{1}\right]=0$ where $C_{1}$ denotes the lefthand side of Eq. (16). Demanding $\dot{\Pi}_{\lambda}=0$ implies the constraint $C_{2} \equiv$ $Z^{\dagger} Z-1=0$ exactly as before. This constraint, however, is not the generator of any obvious symmetry. Indeed, the commutator is not zero but rather $i\left[H, C_{2}\right]=$ $\Pi_{z} Z+Z^{\dagger} \Pi_{z^{\dagger}}$. Requiring the time variation of the right-hand side to vanish then leads to a tertiary constraint. This new constraint also fails to commute with the Hamiltonian and a quartenary constraint ensues. For the $\mathrm{CP}^{1}$ model without the Chern-Simons term, this method was carried through to the end and among the constraints thus generated there are first and second class constraints thus necessitating the use of Dirac brackets. ${ }^{9}$ The resulting commutation relations are no longer canonical. We have not pursued this method to the end. From the above, it is clear that the $C_{2}$ constraint is the complication. In the next section we reformulate the problem in terms of new field variables, abandoning the $\left(Z_{1}, Z_{2}\right)$ variables and using radial field coordinates instead. In the context of work done within the BRST formalism, we will then identify the part of the kinetic Hamiltonian that fails to commute with this constraint and treat it in a new way.

\section{BRST Quantization}

The key insight underlying the use of the BRST method applied to constrained systems as discussed in the NPW paper is to identify the $C_{2}$ constraint as applying to only the radial degree of freedom in field space. This identification allows one to suitably truncate the non-commuting piece from the kinetic part of the Hamiltonian and form a new Hamiltonian density $\mathcal{K}^{\prime}$. From $\mathcal{H}^{\prime}$, _one constructs the "canonical Lagrangian" density $\mathcal{L}^{\prime}$ and after doing so, one identifies the symmetry of the new Lagrangian for which $\lambda(x)$ plays a role similar to the role played by the $A_{0}(x)$ field under time dependent transformations. This new symmetry is then elevated to a BRST symmetry and an appropriate 
gauge-fixing term $\mathcal{L}_{\text {BRST }}$ is added. Finally, one is instructed to calculate the canonical BRST-fixed Hamiltonian $\mathcal{H}_{\text {BRST }}$ which by construction will commute with the BRST and anti-BRST charge of the theory. Moreover, since the BRST charge has the constraints explicitly, states annihilated by the BRST charge are eigenstates of the constraints. Therefore, schematically our progression will be as follows:

$$
\mathcal{L} \rightarrow \mathcal{H} \rightarrow \mathcal{H}^{\prime} \rightarrow \mathcal{L}^{\prime} \rightarrow \mathcal{L}^{\prime}+\mathcal{L}_{\mathrm{BRST}} \rightarrow \mathcal{H}^{\prime}+\mathcal{H}_{\mathrm{BRST}} .
$$

We begin by decomposing the Lagrangian density in (1) in terms of $Z_{1}=$ $\alpha_{1}+i \alpha_{2}$ and $Z_{2}=\alpha_{3}+i \alpha_{4}$ as follows:

$$
\begin{aligned}
\mathcal{L} & =\left(\partial_{\mu} \alpha_{i}\right)\left(\partial^{\mu} \alpha_{i}\right)+A_{\mu} A^{\mu}\left(\alpha_{i} \alpha_{i}\right) \\
& +2 A_{\mu}\left(\alpha_{1} \partial^{\mu} \alpha_{2}-\alpha_{2} \partial^{\mu} \alpha_{1}+\alpha_{3} \partial^{\mu} \alpha_{4}-\alpha_{4} \partial^{\mu} \alpha_{3}\right) \\
& -\lambda\left(\alpha_{i} \alpha_{i}-1\right)+\theta \epsilon_{\mu v \lambda} A^{\mu} \partial^{v} A^{\lambda}
\end{aligned}
$$

While we are still at the Lagrangian level, we will change our field coordinates. The four bosonic field coordinates $\alpha_{i}(x)$ describe $R^{4}$ and the product $\alpha_{i} \alpha_{i}$ is just the radius squared. Thus, constraint $C_{2}$ implies the phase space is restricted to the hypersphere $R \equiv \alpha_{i} \alpha_{i}=1$. There remain three angular degrees of freedom. To capture this completely, we recast the Lagrangian density in radial coordinates making the usual transformations:

$$
\begin{array}{ll}
\alpha_{1}=R \cos \theta & \alpha_{3}=R \sin \theta \sin \phi \cos \psi \\
\alpha_{2}=R \sin \theta \cos \phi & \alpha_{4}=R \sin \theta \sin \phi \sin \psi
\end{array} .
$$

The inverse transformations relate the radial and angular variables to the alphas. The new nonlinear Lagrangian density becomes

$$
\begin{aligned}
\mathcal{L} & =\left(\partial_{\mu} R \partial^{\mu} R\right)+R^{2}\left[\left(\partial_{\mu} \theta \partial^{\mu} \theta\right)+\sin ^{2} \theta\left\{\left(\partial_{\mu} \phi\right)\left(\partial^{\mu} \phi\right)+\sin ^{2} \phi\left(\partial_{\mu} \psi \partial^{\mu} \psi\right)\right\}\right] \\
& +2 R^{2} A_{\mu}\left(\cos \phi \partial^{\mu} \theta-\frac{1}{2} \sin 2 \theta \sin \phi \partial^{\mu} \phi+\sin ^{2} \theta \sin ^{2} \phi \partial^{\mu} \psi\right) \\
& +A_{\mu} A^{\mu} R^{2}+k \varepsilon_{\mu \nu \lambda} A^{\mu} \partial^{\nu} A^{\lambda}-\lambda\left(R^{2}-1\right),
\end{aligned}
$$


where we have used $k$ as the coefficient of the Chern-Simons term instead of $\theta$. The Lagrangian in the old coordinates was invariant with respect to a $\mathrm{U}(1)$ gauge symmetry which is now realized as follows for a gauge function $\eta(x)$

$$
\begin{aligned}
& \delta \theta=\cos \phi \eta \quad \delta \phi=-\cot \theta \sin \phi \eta \quad \delta \psi=\eta \\
& \delta R=0 \quad \delta A_{\mu}=-\partial_{\mu} \eta \quad \delta \lambda=0 .
\end{aligned}
$$

For functions $\eta(x)$ which vanish at infinity, the Lagrangian is invariant as before under the above transformation.

The Hamiltonian formalism is more complex in these new coordinates. The new canonical momenta are

$$
\begin{aligned}
\Pi_{R} & =2 \dot{R} & \Pi_{\psi} & =2 R^{2}\left(\sin ^{2} \theta \sin ^{2} \phi \dot{\psi}+f_{\psi} A_{0}\right) \\
\Pi_{\theta} & =2 R^{2}\left(\dot{\theta}+f_{\theta} A_{0}\right) & \Pi_{\phi} & =2 R^{2}\left(\sin ^{2} \theta \dot{\phi}+f_{\phi} A_{0}\right),
\end{aligned}
$$

where we have defined $f_{\theta}=\cos \phi, f_{\phi}=-\frac{1}{2} \sin 2 \theta \sin \phi$, and $f_{\psi}=\sin ^{2} \theta \sin ^{2} \phi$. Again in adopting the canonical procedure, we find the two primary constraints $\Pi_{A_{0}}=\Pi_{\lambda}=0$. In writing the Hamiltonian density, we will as in Sec. III add a total time derivative to eliminate $A_{2}(x)$ in favor of $\Pi_{A_{1}}(x)$. The resulting Hamiltonian density is

$$
\begin{aligned}
\mathcal{H} & =\frac{1}{4}\left(\Pi_{R}^{2}+\frac{1}{R^{2}}\left(\Pi_{\theta}^{2}+\frac{1}{\sin ^{2} \theta}\left[\Pi_{\phi}^{2}+\frac{1}{\sin ^{2} \phi} \Pi_{\psi}^{2}\right]\right)\right) \\
& -A_{0}\left(\cos \phi \Pi_{\theta}-\cot \theta \sin \phi \Pi_{\phi}+\Pi_{\psi}+\partial_{1} \Pi_{A}-2 k \partial_{2} A\right) \\
& +\mathcal{K}\left(\partial_{i} R, \partial_{i} \theta, A, \Pi_{A}, \cdots\right)+\lambda\left(R^{2}-1\right),
\end{aligned}
$$

where $A$ and $\Pi_{A}$ denote the two gauge degrees of freedom and $\mathcal{K}$ is short for $\left(D_{i} Z\right)^{\dagger}\left(D_{i} Z\right)$ in radial coordinates with $i=1,2$. The transformations of the canonical momenta are read off from their operator definitions and they are

$$
\begin{array}{ll}
\delta \Pi_{\theta}=-\eta \csc ^{2} \theta \sin \phi \Pi_{\phi} & \delta \Pi_{\psi}=0 \\
\delta \Pi_{\phi}=\eta\left(\sin \phi \Pi_{\theta}+\cot \theta \cos \phi \Pi_{\phi}\right) & \delta \Pi_{R}=0
\end{array}
$$

and, as before, $\delta A=-\partial_{1} \eta$ and $\delta \Pi_{A}=-2 k \partial_{2} \eta$. In fact, all of the transformations could be read off by using the "Gauss's Law" constraint multiplying $A_{0}$ in the 
equation for $\mathcal{H}$ which we called $C_{1}$ in Sec. III and is written explicitly below. The variation of an operator $\mathcal{O}$ would then be $\delta O(x)=i\left[\int d^{2} x^{\prime} \eta\left(x^{\prime}\right) \mathcal{C}_{1}\left(x^{\prime}\right), \mathcal{O}(x)\right]$. In computing the commutator, we impose the canonical equal-time commutation relations for the radial and angular variables

$$
\begin{aligned}
{\left[R(x), \Pi_{R}\left(x^{\prime}\right)\right] } & =\left[\theta(x), \Pi_{\theta}\left(x^{\prime}\right)\right]=\left[\phi(x), \Pi_{\phi}\left(x^{\prime}\right)\right] \\
& =\left[\psi(x), \Pi_{\psi}\left(x^{\prime}\right)\right]=i \delta^{2}\left(x-x^{\prime}\right) .
\end{aligned}
$$

Given the transformation rules in Eqs. (20) and (23), we find that $\delta \mathcal{H}=\dot{\eta} C_{1}$. Therefore, as long as physical states are annihilated by the constraint $C_{1}$, this variation is unimportant.

The vanishing of the two primary constraints $\left(\Pi_{\lambda}, \Pi_{A_{0}}\right)$ for all time implies the two secondary constraints $C_{1}=0$ and $C_{2} \equiv R^{2}-1=0$. The vanishing of the secondary constraint $C_{1}$ for all time requires the commutator of $\mathcal{C}_{1}$ with the Hamiltonian to cancel. After arduous manipulations and the use of the fact that the Hamiltonian is normal ordered (as is the constraint), it is confirmed that $\left[H, C_{1}\right]=0$. The cancellations are more evident using a more symmetric expression for the $\mathcal{C}_{1}$ constraint as

$$
C_{1}=\cos \phi \Pi_{\theta}-\frac{1}{2} \cot \theta\left(\sin \phi \Pi_{\phi}+\Pi_{\phi} \sin \phi\right)+\Pi_{\psi}+\partial_{1} \Pi_{A}-2 k \partial_{2} A
$$

Constraint $C_{2}$ fails to commute with the Hamiltonian in presence of the $\Pi_{R}{ }^{2}$ piece. Hence we have isolated the specific kinetic piece that the constraint fails to commute with. In Sec. III, this is what led to a tower of constraints.

The constraint $C_{2}$ thus leads us to write a new Hamiltonian density $\mathcal{H}^{\prime}=$ $\mathcal{H}-\frac{1}{4} \Pi_{R} \cdot{ }^{2}$ This leaves the gauge-invariance properties of the Hamiltonian unaltered since the variations of both the fields $R(x)$ and $\Pi_{R}(x)$ is zero. The new Hamiltonian trivially satisfies $\left[H^{\prime}, C_{2}\right]=0$ ! The equations of motion given by $\dot{O}=i\left[H^{\prime}, 0\right]$ are unchanged except now we have $\dot{R}=0$ whereas before it was $\dot{R}=\Pi_{R}$. This independence of $\dot{R}$ and $\Pi_{R}$ is crucial to constructing a new gauge symmetry among these variables. More precisely, the new 
Hamiltonian is given by $H^{\prime}=\int d^{2} x \bar{\Theta}^{00}$ where $\Theta^{\mu \nu}$ is the canonical energymomentum tensor and $\bar{\Theta}^{00}=\Theta^{00}-\frac{1}{4} \Pi_{R}^{2}$. We note that among the $\Theta^{\mu \nu}$ 's, only the (00) component is shifted. The conserved charges $P^{i}=\int d^{2} x \Theta^{0 i}$ and $M^{i j}=\int d^{2} x\left(x^{i} \Theta^{0 j}-x^{j} \Theta^{0 i}\right)$ are unchanged. However, the conserved charges $M^{0 j}$ become $\bar{M}^{0 j}=\int d^{2} x\left(x^{0} \Theta^{0 j}-x^{j} \bar{\Theta}^{00}\right)$. The barred and unbarred quantities realize the usual Poincare group in $2+1$ space-time dimensions with the exception of two commutators. Using the canonical commutation relations quoted above, the two anomalous commutators are $\left[\bar{M}^{0 j}, H^{\prime}\right]=i\left(P^{j}-P_{R}^{j}\right)$ where $P_{R}^{j}=\int d^{2} x \Theta_{R}^{0 j}=\int d^{2} x \Pi_{R} \partial^{j} R$ and $\left[\bar{M}^{0 j}, \bar{M}^{0 k}\right]=i\left(M^{j k}-M_{R}^{j k}\right)$ where $M_{R}^{j k}=\int d^{2} x\left(x^{j} \Theta_{R}^{0 k}-x^{k} \Theta_{R}^{0 j}\right)$. These anomalous pieces in the charge algebra of the space-time group $I S O(2,1)$ will vanish when sandwiched between any states in the physical subspace of the full Hilbert space. If we can diagonalize the new Hamiltonian and the constraint $\mathcal{C}_{2}$, then the expectation value for physical states $\left\langle\Psi\left|\Theta_{R}^{0 j}\right| \Psi\right\rangle$ is zero since $\Theta_{R}^{0 j} \approx \partial^{j} R$. If this holds for time $t=t_{0}$ then it will be true for all time, since $\left[\Theta_{R}^{0 j}, H^{\prime}\right]=0$. Therefore, in the physical subspace, we recover Lorentz invariance. Using this new Hamiltonian, we delineate how we can indeed exactly define the physical subspace.

With the new Hamiltonian density, we derive the Lagrangian density via the reverse canonical procedure:

$$
\mathcal{L}=\sum_{i}\left(\Pi_{\chi} \dot{\chi}\right)-\not H
$$

where $\chi$ stands for the fields $R, \theta, \phi, \psi, A$ and $A_{0}$. The $\lambda$ field has been purposely left out in order that the resulting Lagrangian have a new gauge symmetry. The resulting Lagrangian density is

$$
\mathcal{L}^{\prime}=\Pi_{R} \dot{R}+\Pi_{\theta} \dot{\theta}+\Pi_{\phi} \dot{\phi}+\Pi_{\psi} \dot{\psi}+\Pi_{A} \dot{A}+\Pi_{A_{0}} \dot{A}_{0}-\mathscr{H}^{\prime}
$$

It is easy to check using the transformation rules for the fields and their canonically conjugate momenta that $\mathcal{L}^{\prime}$ has the $U(1)$ gauge invariance as before. Now we are in a position to unravel the additional symmetry of this new Lagrangian. 
The fields $R, \Pi_{R}$ and $\lambda$ were invariant with respect to the usual gauged $\mathrm{U}(1)$. Concentrating on these fields only, the Lagrangian density has the form $\Pi_{R} \dot{R}-$ $\lambda\left(R^{2}-1\right)$. The phase space should be constrained to the same hypersphere whether the constraint reads $R^{2}=1$ or $R=1$ since the latter certainly implies the former. From now on, we will by fiat write the constraint as $\lambda(R-1)$ which is equivalent to starting from a $C P^{1}$ defined with a constraint $\lambda\left(\sqrt{Z^{\dagger} Z}-1\right)$ in the original Lagrangian. The point of treating the constraint in this way lies in a new gauge invariance embedded in $\Pi_{R} \dot{R}-\lambda(R-1)$ given by

$$
\delta R=0 \quad \delta \Pi_{R}=\rho \quad \delta \lambda=-\dot{\rho},
$$

under which $\delta \mathcal{L}^{\prime}=\frac{d}{d t}[\rho(R-1)]$. Since it is a total time derivative, it will not affect the physics. The above variations reveal again the independence of $\dot{R}$ and $\Pi_{R}$.

Now we have two symmetries and we can use the BRST method to arrive at the gauge fixed Lagrangian. The resulting Lagrangian will give the final Hamiltonian and the invariance properties of the BRST gauge fixed Lagrangian will be expressed as the commutation of the BRST charge with the Hamiltonian. This in turn, will mean all kets annihilated by the BRST charge will automatically obey the constraint and the gauge fixing condition. The BRST gauge symmetry is realized by elevating the gauge parameter functions to anti-commuting "ghost" fields that close in such a way that twice the variation of any quantity is zero. Specifically, the BRST transformation corresponding to the $\rho$ parameter becomes

$$
\delta \Pi_{R}=c_{2} \quad \delta \lambda=-\dot{c}_{2} \quad \delta c_{2}=0 \quad \delta \bar{c}_{2}=b_{2} \quad \delta b_{2}=0
$$

where $c_{2}$ and $\bar{c}_{2}$ are the ghost and anti-ghost fields with subscript two corresponding to the two in $C_{2}$. The variation of all other fields is zero under this BRST symmetry. The second BRST transformation corresponds to the $\eta$ parameter 


$$
\begin{array}{rlrl}
\delta \theta & =\cos \phi c_{1} & \delta \Pi_{\theta} & =-c_{1} \csc ^{2} \theta \sin \phi \Pi_{\phi} \\
\delta \phi & =-\cot \theta \sin \phi c_{1} & \delta \Pi_{\phi} & =c_{1}\left(\sin \phi \Pi_{\theta}+\cot \theta \cos \phi \Pi_{\phi}\right) \\
\delta \psi & =c_{1} & \delta A_{0} & =-\dot{c_{1}} \\
\delta A & =-\partial_{1} c_{1} & \delta \Pi_{A} & =-2 k \partial_{2} c_{1} \\
\delta c_{1} & =0 & \delta \bar{c}_{1} & =b_{1} \\
\delta b_{1} & =0 &
\end{array}
$$

and all other variations vanishing. We see that the two symmetries are orthogonal to each other, that is, each acts on a different set of field from the Lagrangian. In order to gauge fix the new symmetry, we add to $\mathcal{L}^{\prime}$ the following $\delta \mathcal{L}_{1}$ :

$$
\delta \mathcal{L}_{1} \equiv-\delta\left[\bar{c}_{2}\left(\dot{\lambda}-\Pi_{R}+\frac{1}{2} b_{2}\right)\right]
$$

Now we have the freedom to gauge fix the usual $U(1)$ gauge symmetry for which we will choose $\partial_{\mu} A^{\mu}=0$ which in our variables corresponds to $\dot{A}_{0}-\partial_{1} A-$ $\frac{1}{2 k} \partial_{2} \Pi_{A}=0$. The appropriate gauge fixing term in the BRST language is

$$
\delta \mathcal{L}_{2} \equiv-\delta\left[\bar{c}_{1}\left(\dot{A}_{0}-\partial_{1} A-\frac{1}{2 k} \partial_{2} \Pi_{A}+\frac{1}{2} b_{1}\right)\right]
$$

Using the BRST transformations given above and writing the gauge condition for the $A(x)$ field as $G(x)$, these two terms can be expanded and summed giving

$$
\begin{aligned}
\delta L_{\mathrm{BRST}} & =-b_{1} G(x)-\frac{1}{2}{b_{1}}^{2}+\dot{\bar{c}}_{1} \dot{c}_{1}-\partial_{i} \bar{c}_{1} \partial_{i} c_{1} \\
& +-b_{2}\left(\dot{\lambda}-\Pi_{R}\right)-\frac{1}{2}{b_{2}}^{2}+\dot{\bar{c}}_{2} \dot{c}_{2}-\bar{c}_{2} c_{2}
\end{aligned}
$$

where we have added a total time derivative for the ghost fields and performed a partial integration for the last term. This makes the total Lagrangian $\mathcal{L}_{\mathrm{CP}}=$ $\mathcal{L}^{\prime}+\delta \mathcal{L}_{\text {BRST }}$ 
Given this new Lagrangian, we form again the canonical momenta and the Hamiltonian. The new canonical momenta resulting from the BRST term are $\Pi_{\lambda}=-b_{2}, \Pi_{A_{0}}=-b_{1}, \Pi_{c_{i}}=\dot{\bar{c}}_{i}, \Pi_{\bar{c}_{i}}=\dot{c}_{i}$. The first two equations imply we can eliminate the $b(x)$ degrees of freedom in favor of the relative $\Pi(x)$ degrees of freedom. Therefore, we can rewrite $\delta L_{\mathrm{BRST}}$

$$
\delta L_{\mathrm{BRST}}=\Pi_{\lambda} \dot{\lambda}+\Pi_{A_{0}} \dot{A}_{0}+\Pi_{c_{1}} \dot{c_{1}}+\dot{\overline{c_{1}}} \Pi_{\bar{c}_{1}}+\Pi_{c_{2}} \dot{c_{2}}+\dot{\overline{c_{2}} \Pi}-\delta \mathcal{H}_{\mathrm{BRST}}
$$

with $\mathfrak{K}_{\text {BRST }}$ given by

$$
\begin{aligned}
\delta \mathcal{H}_{\mathrm{BRST}} & =\dot{\bar{c}_{1}} \dot{c}_{1}+\dot{\bar{c}_{2}} \dot{c}_{2}+\bar{c}_{2} c_{2}+\partial_{i} \bar{c}_{1} \partial_{i} c_{1} \\
& +\Pi_{\lambda} \Pi_{R}+\frac{1}{2} \Pi_{\lambda}{ }^{2}+\Pi_{A_{0}}\left(\partial_{1} A+\frac{1}{2 k} \partial_{2} \Pi_{A}\right)+\frac{1}{2} \Pi_{A_{0}}{ }^{2},
\end{aligned}
$$

where we have used time derivatives instead of canonical momenta for the ghost fields to make the symmetries more familiar. Combining these terms with the expression relating $\mathcal{L}^{\prime}$ and $\mathcal{H}^{\prime}$ in Eq. (24), we read off the final Hamiltonian $H_{\mathrm{CP}}=\mathfrak{H}^{\prime}+\delta \mathcal{H}_{\mathrm{BRST}}$.

The construction of the BRST and anti-BRST charge operators is straightforward. We require of the BRST charge when acting on a field $\Phi(x)$ to produce the same variation as given in Eqs. (27) and (28) using $[Q, \Phi]$ for bosonic degrees of freedom and $\{Q, \Phi\}$ for fermionic degrees of freedom. The anti-BRST charge on the other hand should yield a variation with the parameter $-\bar{c}$ instead of $c$. The two BRST charges and the anti-BRST charges are given by

$$
\begin{aligned}
& Q_{1}=-i \int d^{2} x\left[-c_{1} c_{1}+\dot{c}_{1} \Pi_{A_{0}}\right] \\
& \bar{Q}_{1}=i \int d^{2} x\left[-\bar{c}_{1} \mathcal{C}_{1}+\dot{\bar{c}_{1}} \Pi_{A_{0}}\right] \\
& Q_{2}=-i \int d^{2} x\left[c_{2}(R-1)+\dot{c}_{2} \Pi_{\lambda}\right] \\
& \bar{Q}_{2}=i \int d^{2} x\left[\bar{c}_{2}(R-1)+\dot{\bar{c}_{2}} \Pi_{\lambda}\right] .
\end{aligned}
$$

With the canonical commutation relations $\left[\lambda(x), \Pi_{\lambda}\left(x^{\prime}\right)\right]=\left[R(x), \Pi_{R}\left(x^{\prime}\right)\right]=$ $\left[A_{0}(x), \Pi_{A_{0}}\left(x^{\prime}\right)\right]=i \delta^{2}\left(x-x^{\prime}\right)$ and the anticommutation relations $\left\{c_{i}(x), \dot{\bar{c}}_{i}\left(x^{\prime}\right)\right\}=$ 
$-\left\{\bar{c}_{i}(x), \dot{c}_{i}\left(x^{\prime}\right)\right\}=i \delta^{2}\left(x-x^{\prime}\right)$, it can easily verified that the correct transformations are reproduced and the relations $\left[Q_{i}, H_{\mathrm{CP}}\right]=\left[\bar{Q}_{i}, H_{\mathrm{CP}}\right]=0$ are satisfied. The single non-canonical anticommutation relation between the anti-ghost and its momenta means with the minus sign, states with any number of anti-ghosts will have negative norm. Since the ghosts do not interact with the physical fields, our physical states will always remain in the positive norm subspace in the Hilbert space of states. A further discussion of this is found in the NPW paper. Finally, we write down the final quantum Hamiltonian for our constrained system:

$$
\begin{aligned}
\mathcal{H}_{\mathrm{CP}} & =\frac{1}{4 R^{2}}\left[\Pi_{\theta}{ }^{2}+\frac{1}{\sin ^{2} \theta}\left\{\Pi_{\phi}{ }^{2}+\frac{1}{\sin ^{2} \phi} \Pi_{\psi}{ }^{2}\right\}\right] \\
& +\left(\partial_{i} R \partial_{i} R\right)+R^{2}\left[\left(\partial_{i} \theta \partial_{i} \theta\right)+\sin ^{2} \theta\left\{\left(\partial_{i} \phi\right)\left(\partial_{i} \phi\right)+\sin ^{2} \phi\left(\partial_{i} \psi \partial_{i} \psi\right)\right\}\right] \\
& +2 R^{2} A_{i}\left(\cos \phi \partial_{i} \theta-\frac{1}{2} \sin 2 \theta \sin \phi \partial_{i} \phi+\sin ^{2} \theta \sin ^{2} \phi \partial_{i} \psi\right)+A_{i} A_{i} R^{2} \\
& -A_{0}\left(\cos \phi \Pi_{\theta}-\cot \theta \sin \phi \Pi_{\phi}+\Pi_{\psi}+\partial_{1} \Pi_{A}-2 k \partial_{2} A\right)+\lambda(R-1) \\
& +\dot{\bar{c}}_{1} \dot{c}_{1}+\dot{\bar{c}}_{2} \dot{c}_{2}+\bar{c}_{2} c_{2}+\partial_{i} \bar{c}_{1} \partial_{i} c_{1} \\
& +\Pi_{\lambda} \Pi_{R}+\frac{1}{2} \Pi_{\lambda}{ }^{2}+\Pi_{A_{0}}\left(\partial_{1} A+\frac{1}{2 k} \partial_{2} \Pi_{A}\right)+\frac{1}{2} \Pi_{A_{0}}{ }^{2}
\end{aligned}
$$

where $A_{2}(x)$ in the third line is by definition $\frac{1}{2 k} \Pi_{A}$. The equations $Q_{i}|\Psi\rangle=$ $\bar{Q}_{i}|\Psi\rangle=0$ define the physical states as the BRST invariant states. Such states exactly satisfy $C_{1}|\Psi\rangle=C_{2}|\Psi\rangle=\Pi_{\lambda}|\Psi\rangle=\Pi_{A_{0}}|\Psi\rangle=0$. When these operator equations are substituted into $\mathcal{H}_{C P}$ we arrive at the Hamiltonian acting on the physical states of the theory. This completes the quantization of the $\mathrm{CP}^{1}$ model with Chern-Simons term. In a previous paper, the author found models isomorphic to this model without the nefarious constraint $R=1$. It has an implicit systematic expansion that exactly realizes the constraint similar to chiral Lagrangians. The BRST quantization of this model does not require the intro-duction of $\Pi_{R}$ ever in the Lagrangian as a separate degree of freedom and hence the BRST fixed Lagrangian can be directly used in the path integral formulation of the theory. We turn our attention now to this easier problem. 


\section{BRST Gauge Fixing of the SU(2) Model}

The $C P^{1}$ model can be viewed as a theory of four real scalar fields constrained to a modulus of one with a gauge invariant interaction. The addition of the Chern-Simons term gives the two spatial gauge degrees of freedom dynamics like a kinetic term would. Unlike the usual $F_{\mu \nu} F^{\mu \nu}$ term, the Chern-Simons term constrains the $A_{2}$ field to be the canonically conjugate momentum of the $A_{1}$ field. This non-trivial observation makes this model worthy of study. One expects ${ }^{10}$ higher loop effects to contribute the usual kinetic piece to the effective action and thus change the dynamics. It would be valuable though to fully understand the lowest order theory in order to systematically derive all of the higher dimensional operators in the effective action. The notion of four scalar fields constrained is reminiscent of pion theory in the limit of large $m_{\sigma}$, viz chiral Lagrangians. In fact, this analogy can be made exact by writing the four component fields $\alpha_{i}$ by defining a matrix $U(x)$ as

$$
U=\alpha_{1}+i \alpha_{i} \sigma^{i}
$$

where the $\sigma$ 's are the usual Pauli $2 \times 2$ matrices. The constraint $\alpha_{i} \alpha^{i}=1$ translates to $U^{\dagger} U=1$, i.e., the special unitary matrices. Note that $\operatorname{det}(U)=\alpha_{i} \alpha^{i}$. Parametrizing $U$ as $U=e^{i \vec{\theta}(x) \cdot \vec{\sigma}}$, we have replaced the four $\alpha$ 's by three $\theta$ degrees of freedom. Hence, if we stay in the Hilbert space of $\theta$ 's, we are exactly at the constraint.

The resulting Lagrangian density in the $U(x)$ formalism using an equivalent map as that in Eq. (36) is ${ }^{11}$

$$
\mathcal{L}=\frac{1}{2} \operatorname{Tr}\left[\left(D_{\mu} U\right)^{\dagger}\left(D^{\mu} U\right)\right]+k \varepsilon^{\mu \nu \lambda} A_{\mu} \partial_{\nu} A_{\lambda}
$$

with $D_{\mu} U=\left(\partial_{\mu}+i A_{\mu} \sigma_{z}\right) U$. From now on, we are instructed to think of $U(x)$ in terms of an expansion in $\theta(x)$ which means that the above Lagrangian density has an infinite number of interactions. What is the analog of $f_{\pi}$, the expansion 
parameter for chiral Lagrangians? In the $\mathrm{CP}^{1}$ model, if the $Z(x)$ are dimensionless, there will appear a dimension-full parameter in front of the lowest order action. This parameter will serve as $f_{\pi}$.

In quantizing the model, we will encounter the usual "Gauss's" law (secondary) constraint which we called $C_{1}$ in previous sections. It commuted with the Hamiltonian and presented no further constraints. The origin of this was the lack of a canonical conjugate field momentum for $A_{0}$. The constraint must generate the following gauge variations:

$$
\begin{gathered}
\delta U=i \eta \sigma_{z} U \\
\delta A_{\mu}=-\partial_{\mu} \eta
\end{gathered}
$$

If the IIamiltonian formalism is carried through in the framework of $U_{i j}$ 's as the above variations exhibit, the resulting progression will be similar to the exercise with the $\alpha$ 's $\left(\alpha_{i} \sim U_{k l}\right)$ and a constraint on the modulus of the $U_{i j}$ will have to be added. Therefore, one must instead expand the $U$ fields in terms of the $\theta$ fields and then proceed. This expansion can be truncated to the most significant lowest order terms and then quantized. Having described this, we will use the BRST method to gauge fix the action and define the path integral for an alternative formulation of the perturbation theory for this model.

The Feynman path integral with the above Lagrangian will be divergent in view of the $\mathrm{U}(1)$ gauge invariance of the theory. We need to add an appropriate gauge fixing piece. The BRST gauge fixing procedure introduces an anti-commuting ghost and anti-ghost field and one additional bose field, the $b(x)$ field. Integration over this last field will introduce the appropriate symmetry breaking term to define the path integral properly. An alternative route is the Fadeev-Popov procedure. For the gauge $\partial_{\mu} A^{\mu}=0$, the appropriate BRST term -was given in a previous section:

$$
\delta \mathcal{L}_{\mathrm{BRST}}=-b\left(\partial_{\mu} A^{\mu}\right)-\frac{1}{2} b^{2}+\partial_{\mu} \bar{c} \partial^{\mu} c
$$


where we have performed partial integrations for the ghost fields. The BRST transformations for the ghost fields were given in Eq. (28). The others are given in Eq. (38), thus $\delta U=i c \sigma_{z} U$ to lowest order in $\theta$ becomes $\delta \theta_{z}=i c, \delta \theta_{x}=i c \theta_{y}$ and $\delta \theta_{y}=-i c \theta_{x}$. The path integral over the $b(x)$ is just a Gaussian integral giving (const) $e^{\frac{1}{2}\left(\partial_{\mu} A^{\mu}\right)^{2}}$. This is exactly the gauge fixing piece that one gets from the Fadeev-Popov procedure. We see that the ghosts do not interact with the matter fields and so they can be thrown out as one does in QED for the path integral. Thus, BRST gauge fixing introduces the usual gauge fixing piece and the final Lagrangian is

$$
\mathcal{L}=\frac{1}{2} \operatorname{Tr}\left[\left(D_{\mu} U\right)^{\dagger}\left(D^{\mu} U\right)\right]+\frac{1}{2}\left(\partial_{\mu} A^{\mu}\right)^{2}+k \varepsilon^{\mu \nu \lambda} A_{\mu} \partial_{\nu} A_{\lambda}
$$

where by $U$ we mean $U\left(\theta_{i}\right)$. The integrations for the Feynman amplitude have to be done over $\theta_{i}$ and $A_{\mu}$. It has been shown using the path integral method that the bose degrees of freedom have fermi-like propagators and vice versa in the low momentum regime. It would be interesting to show with equal rigor the transmutations for the solitons ${ }^{12}$ of this model.

\section{Conclusions and Remarks}

In the framework of classical Lagrangian analysis, it was shown how all the gauge degrees of freedom could be written in terms of the $\mathrm{CP}^{1}$ fields with various non-local interactions. Quantizing the theory traditionally meant a horrendous set of constraints which leads to non-canonical commutation relations and a physical Hilbert space that is difficult to grasp. At the cost of using a nonlinear Lagrangian and introducing new propogating degrees of freedom within the _framework of BRST as elaborated in the NPW paper, we were able to quantize the theory and come to terms with the set of physical states. Besides the larger number of degrees of freedom for our model, the entire quantization is similar to the problem of the rotor in the NPW paper. The BRST method was applied then 
to a model isomorphic to the $\mathrm{CP}^{1}$ model to obtain a path-integral action without a modulus constraint and with the gauge fixed. Presumably, since the ghosts do not interact with the gauge fields, no spin statistics transmutation should occur for them. It is also not clear how to show rigorously that the soliton excitations will be transmuted. The model studied has a curious kinetic piece for the gauge fields making the two spatial gauge degrees of freedom canonical conjugates of each other. It would be amusing to compute the lowest order corrections to the propagator for the gauge degree of freedom. Although the $\mathrm{CP}^{N}$ model in two dimensions has been extensively studied, this model in $2+1$ space-time dimensions and particularly with the Chern-Simons term has only recently received genuine interest.

\section{Acknowledgements}

The author would like to thank Dieter Issler for many fruitful discussions and a critical reading, Roger Brooks for pointing out the work of Bowick et al., Lance Dixon for a crucial remark in Sec. III, and Sebastian Doniach for his constant support. 


\section{References}

1. H. Eichenherr, Nucl. Phys. B146 (1978) 215; B155 (1979) 544; V. Golo and A. Perelemov, Phys. Lett. 79B (1978) 112.

2. F. Wilczek and A. Zee, Phys. Rev. Lett. 51 (1983) 2250; A. M. Polyakov, Mod. Phys. Lett. A3 417; A. M. Din and W. J. Zakrzewski, Phys. Lett. 146B (1984) 341; T. Jacobsen, Boston University preprint BUHEP-88-12;

A. Dhar, Tata Institute preprint TIFR/TH/88-36; G. Semenoff, Print88-0386; Y. Wu and A. Zee, Phys. Lett. 147B (1984) 325; M. Bowick, D. Karabali and L. C. R. Wijewardhana, Nucl. Phys. B 271 (1986) 417; C. Tze, Mod. Phys. Lett. A3 (1988) 1959.

3. I. Dzyaloshinskii, A. Polyakov and P. Wiegmann, Phys. Lett. A127 (1988) 112; P. Wiegmann, Phys. Rev. Lett. 60 (1988) 821.

4. K. Karabali and G. Murthy, Phys. Rev. D35 (1987) 1522; J. Maharana, Phys. Lett. 128B (1983) 411; M. Bowick et al., loc. cit.

5. E. Gozzi and A. Guha, J. Math. Phys. 24 (1983) 1213.

6. P. A. M. Dirac, Canad. J. Math. 2 (1950) 129.

7. C. Becchi, A. Rouet and R. Stora, Phys. Lett. 52B (1974) 344; Ann. Phys. 98 (1976) 287; Comm. Math. Phys. 42 (1975) 127.

8. D. Nemeschansky, C. Preitschopf and M. Weinstein, SLAC-PUB-4422, 1987 (to be published).

9. E. Gozzi and A. Guha, loc. cit.

10. A. M. Polyakov, Gauge Fields and Strings, Harwood Academic Publishers, (1987) 140.

11. P. Voruganti, SLAC-PUB-4674 (to be published).

12. R. Rajaraman, Solitons and Instantons, North-Holland (1982) 123. 